Reprod. Nutr. Dévelop., 1982, 22 (1 B), 251-260.

\title{
Muscle changes during reinnervation after repeated nerve injuries
}

\author{
J.-C. MIRA
}

Laboratoire de Cytologie, Université P.-et-M. Curie,

7. Quai Saint-Bernard, 75230 Paris Cedex 05

Summary. - Localized freezings of adult rat sciatic nerve, repeated 2 to 5 times at monthly intervals, induced a series of profound changes in the gastrocnemius muscle. In particular, many type IIC fibres were observed one month after the last freezing. However, this muscle transformation was not stable. Our combined cytological, cytochemical and electrophysiological results provide evidence for multiple and polyneuronal innervation of these type IIC fibres.

\section{Introduction.}

Since the many investigations of peripheral nerve regeneration, muscle changes during reinnervation and the properties of reinnervation itself, relatively few studies have been devoted to regeneration and reinnervation after repeated nerve lesions (Mira, 1979 ; Mira and Fardeau, 1978 b). In addition, no attempts have been made to collect and correlate these observations.

In this report, we have therefore tried to correlate the changes in the muscle fibre histochemical properties with the electrophysiological events occurring at the motor endplate during reinnervation.

In collaboration with Michel Fardeau, we applied cytochemical techniques to study the time-course of the changes in muscle fibre type-grouping. Concomitantly, in collaboration with Monique Pécot-Dechavassine, we used electrophysiological techniques to examine the properties of the reinnervated motor endplates.

\section{Material and methods.}

We carried out repeated localized freezings in situ of the left sciatic nerve of adult rats. Freezings were done every 3 weeks to ensure that muscle reinnervation had begun before the nerve was injured again (Mira, 1979). Cold injuries were performed in two different ways. In group I, the sciatic nerve was frozen 1 to 5 times and the results were recorded one month after the last nerve lesion; in 
group II, the test period lasted for several months after the third and final freezing.

Muscle changes were investigated in the medial head of the gastrocnemius muscle at standardized levels (Mira and Fardeau, 1978 a,b). Cryostat serial transverse sections were studied with conventional histo-enzymological techniques (Fardeau, 1973). Fibre types were identified by their myosin-adenosine triphosphate (ATPase) activity according to the classification proposed by Brooke and Kaiser (1970).

A standard intracellular electrophysiological apparatus was used to record endplate potentials (epp's) in surface fibres of the " white » portion of the medial head of the gastrocnemius muscle (Mira and Pécot-Dechavassine, 1978, 1979). The muscles were examined at intervals between 16 and 60 days after the third and final freezing.

\section{Results.}

\section{Muscle changes.}

Normal muscle. - Macroscopically, two well-defined zones were visualized in transverse sections of the gastrocnemius muscle (fig. 1 a) : a crescent-shaped " white " area surrounding most of a " red" area in contact with the aponeurosis.

In cryostat transverse sections, study of the myosin-ATPase activity was used to individualize three principal muscle fibre types (I, IIA and IIB) and a few very rare type IIC fibres. Type I fibres, slightly stained at $\mathrm{pH} \mathrm{9.4,} \mathrm{were} \mathrm{considered}$ as acid-resistant since they became black after pre-incubation at $\mathrm{pH} 4.53$ and 4.35. Type IIA fibres, markedly stained at $\mathrm{pH} 9.4$, were considered as acidsensitive since their ATPase activity was inhibited after pre-incubation at $\mathrm{pH} 4.53$ and 4.35. Type IIB fibres were as dark as type I fibres at $\mathrm{pH} 9.4$ and, although their ATPase activity was completely inhibited after pre-incubation at $\mathrm{pH} 4.35$, they were incompletely inhibited after pre-incubation at $\mathrm{pH} 4.53$. Type IIC fibres were virtually absent from the normal muscle of adult rat; however, when present, they were in contact with type I fibres and, although markedly stained at $\mathrm{pH} 9.4$ (as acid-sensitive fibres), they became partly negative after pre-incubation at $\mathrm{pH} 4.53$ and 4.35 (almost as acid-resistant fibres).

These various muscle fibre types were not equally distributed throughout the muscle transverse section. The red zone contained all the type I fibres, isolated from each other and randomly distributed among fibres mostly of type IIB. The white zone contained only randomly distributed type IIA and IIB fibres.

Silver impregnation showed that each muscle fibre was innervated by a

FIG. 1. - Medial head of rat gastrocnemius muscle. Cryostat transverse sections showing myofibrillar ATPase activity after pre-incubation at $\mathrm{pH} 4.53$ (b) or 4.35 (c, d) and motor terminal innervation after silver impregnation (d).

a : control muscle ; b, c, d : 28, 190 and 20 days after a single localized freezing of the sciatic nerve $(a, b, c: \times 10 ; d: \times 500)$. 


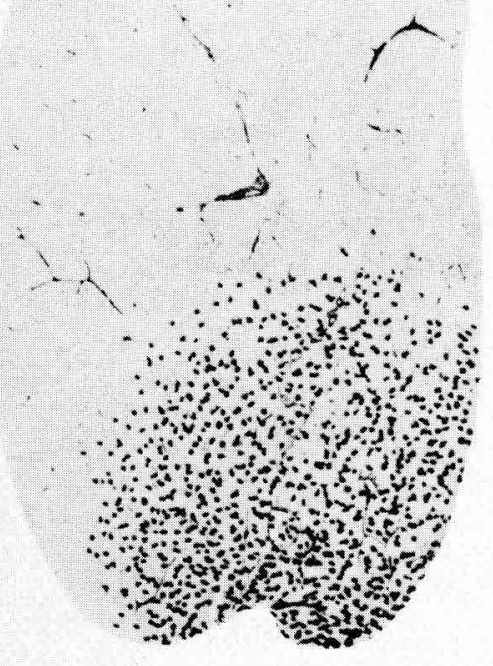

a

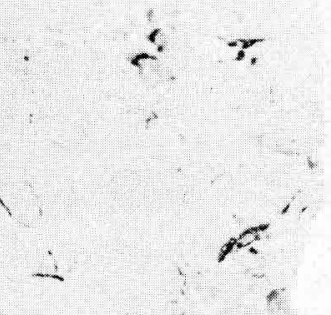

$$
\begin{aligned}
& +2 \text { a }
\end{aligned}
$$

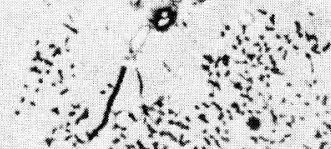

$$
\begin{aligned}
& \because \quad 3 \quad 2 \\
& \because \quad 3 \times \text { r }
\end{aligned}
$$

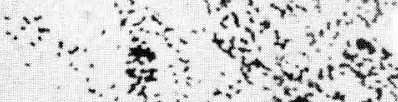

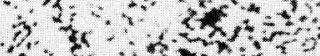

$$
\begin{aligned}
& \therefore \text { स } 3
\end{aligned}
$$

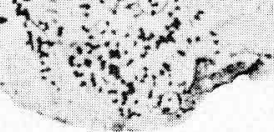


single motor axon which, when it came in contact with the muscle fibre, divided into 2 or 3 fine terminal branches.

Group I. - One month after a single localized freezing of the sciatic nerve, the gastrocnemius muscle exhibited a very limited grouping of fibres of the same histochemical type (fig. 1 b). One month after 2 to 5 freezings, the changes were more pronounced (fig. $2 \mathrm{a}, \mathrm{b}$ ) and there was gradual increase in the number of acid-resistant fibres. Serial sections showed that they belonged to types $I$ and IIC ; in addition, their diameter did not significantly differ from that of the controls. Type II (A or B) fibres, only observed in a small crescent-shaped area at the muscle periphery, were markedly atrophic ; furthermore, like type I fibres, they retained their original cytochemical characteristics.

Group /I. - One month after the third and final freezing, most of the muscle transverse section was occupied by type I and IIC fibres (fig. 2 b). During the second month, the type IIC fibres entirely disappeared (fig. $2 \mathrm{c}$ ) and the diameters of type II (A or B) fibres returned to subnormal values. Only restricted grouping of the different muscle fibre types persisted, and the muscle histochemical pattern did not differ from that observed after a single freezing (fig. $1 \mathrm{c}$ ) ; the situation was the same 3, 6, 12 and 18 months later.

\section{Electrophysiological changes at motor endplates.}

In normal gastrocnemius muscles, all the epp's recorded were simple and the muscle fibres exhibited superimposed and similarly delayed epp's, whatever the intensity of stimulation (fig. 3 a).

In experimental muscles, our investigations were confined to rats which had undergone three localized freezings because nerve and muscle changes had been observed earlier to increase gradually in amplitude between the first and third freezing and to remain stable after further nerve injuries (Mira, 1979; Mira and Fardeau, 1978 a, b). Reinnervation began between 16 and 18 days after the third cold injury; at that time, all the epp's recorded displayed a single peak for all degrees of stimulation, as in normal muscle. Between 20 and 35 days, 15 to 35 p. 100 of the epp's (mean value : 23 p. 100) had two components of different latencies after a single stimulus (fig. $3 \mathrm{~b}, \mathrm{c}, \mathrm{d}$ ) and, in rare cases, three or even four components (fig. 3 e). After 35 days, all the epp's were simple again, as in normal muscle.

Under our experimental conditions, reinnervation of the gastrocnemius muscle was therefore completed in 3 stages: (i) a first stage of simple innervation, starting around day 17 when all the epp's were simple and the

FIG. 2. - Medial head of rat gastrocnemius muscle. Cryostat transverse sections showing myofibrillar ATPase activity after pre-incubation at $p H 4.53(\mathrm{a}, \mathrm{b}, \mathrm{c})$ and motor terminal innervation after silver impregnation (d).

$a, b$ : one month after the sciatic nerve had been frozen two (a) or three (b) times; $c$ : three months after the third and final freezing ; $d: 25$ days after the third and final freezing $(a, b, c): \times 10 ;$ $d: \times 500)$. 
muscle fibres were consequently innervated by a single nerve terminal ; (ii) a stage of multiple innervation (days 20 to 35), during which 23 p. 100 of fibres in each muscle exhibited compound epp's, indicating their innervation by more than one axon. Such type of innervation was even more evident since each epp component usually exhibited different latencies. The compound epp's resulted from the activity of two or several axons contacting the muscle in a close vicinity, because the rise-time of each epp component was similar, and silver impregnation showed several axons converging on the same endplate (fig. $2 \mathrm{~d}$ ). The different delays for each epp component suggest that the axons had different conduction velocities, probably because they were at various stages of maturation ; (iii) a final stage of simple innervation, after 35 days, when all the epp's were simple, indicating that muscle fibres were reinnervated by a single axon.

\section{Discussion.}

The technique of localized freezing was chosen for investigating cytochemical and electrophysiological changes in skeletal muscle during reinnervation because its results are perfectly reproducible. This method also has the advantage of focally destroying all the axons while maintaining the anatomical continuity of the nerve trunk and preserving the basal laminae (Mira, 1971).

The considerable cytochemical and topographical changes observed in the gastrocnemius muscle led us to question the mechanism governing the progressive invasion of almost the entire muscle by a majority of type IIC fibres that are virtually absent from the normal adult rat muscle. According to Brooke et al. (1971), the type IIC corresponds to an undefined type observed during development or reinnervation of adult muscle fibres. It is usually considered as a transitional stage during the conversion of one type into another.

If this concept is true, the above phenomena could be interpreted as a result of a fast regeneration rate for axons belonging to type I motoneurons which normally innervate type I muscle fibres (type I axons), since the latter recover a subnormal volume much faster than type II fibres which remain atrophic but retain their initial cytochemical characteristics. Since the muscle fibres of the same motor unit are known to have similar cytochemical properties (Brooke et al., 1971 ; Burke et al., 1971 ; Edström and Kugelberg, 1968) and to retain their native myosin-ATPase activity during denervation atrophy, any change in their innervation alters their enzymological characteristics. In addition, a new topographical distribution of the diffrerent muscle fibre types, and even the appearance of new types, have been observed in certain adult muscles after

FIG. 3. - Intracellular recordings of endplate potentials in the medial head of the gastrocnemius muscle after three localized freezings of the sciatic nerve. D-tubocurarine 1-3.10-7 $\mathrm{M}$.

a : simple innervation ; b, c, d, e : multiple innervation. Double innervation 19 days (b) and 23 days (c, d) after the third cold injury to the nerve. Triple innervation 19 days after the third freezing (e).

Calibrations : depolarization : $1 \mathrm{mV}$; stimulus : 2 volts ; time : $5 \mathrm{msec}$. 


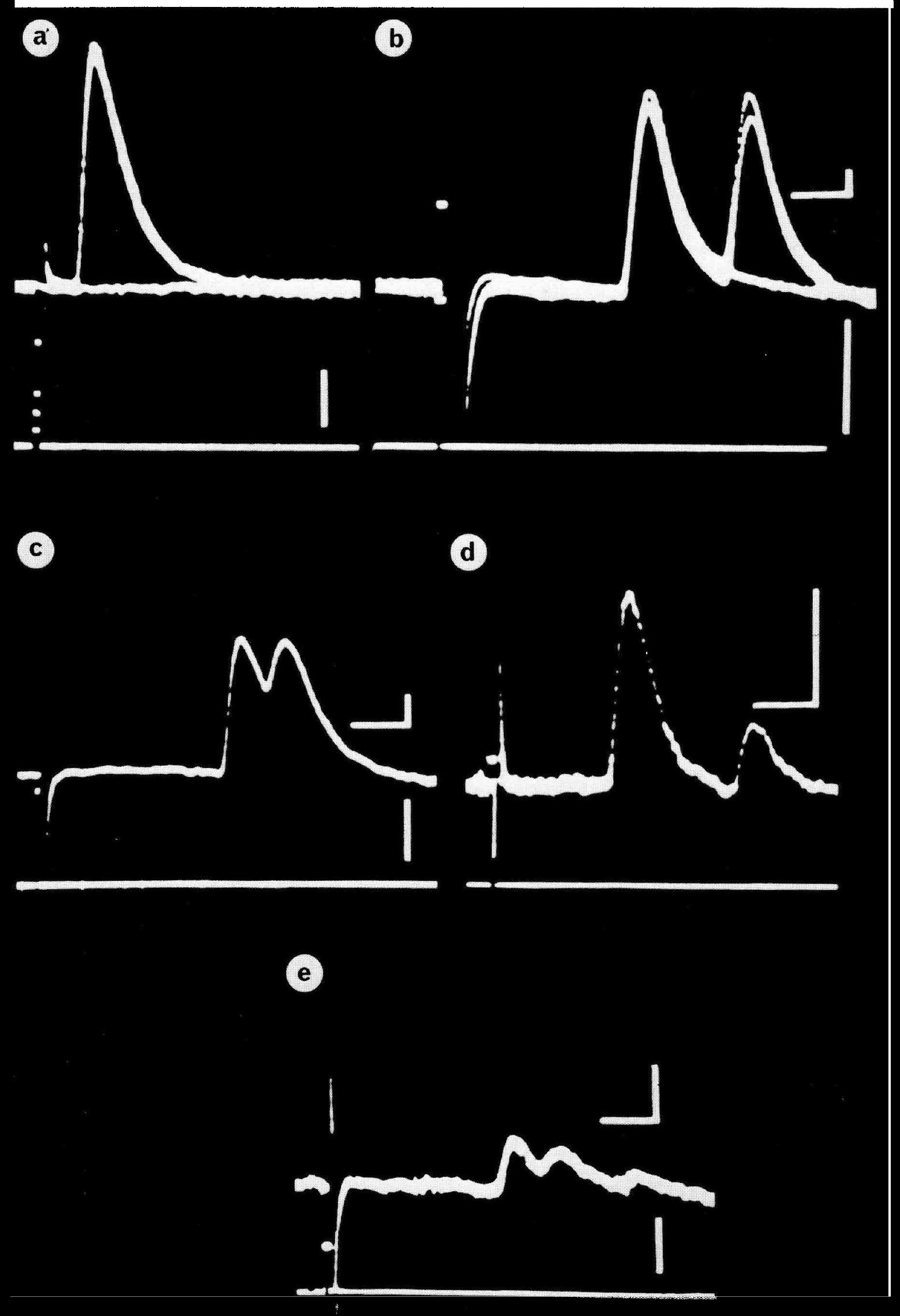


reinnervation. These changes have been interpreted as resulting from the reinnervation of vacant endplates by collateral sprouting of the first regenerated axons (Brooke et al., 1971 ; Karpati and Engel, 1968).

If this is so, type I axons must reinnervate, by collateral sprouting, not only their original type I muscle fibres, but also some contiguous type II fibres. Three of our observations support this hypothesis : (i) after repeated freezings, type I fibres were no longer distributed at random but were grouped in clusters, (ii) type IIC fibres were always localized close to type I fibres, and (iii) after silver impregnation, reinnervation of several contiguous muscle fibres by the same axon was observed (fig. $1 \mathrm{~d}$ ).

Another question was the fate of the very numerous type IIC fibres, virtually absent from the normal muscle. We therefore constituted group II in which the sciatic nerve was locally frozen three times and muscles examined 1 to 18 months later. From the concept that type IIC fibres represent an intermediate stage in the conversion of one cytochemical type into another, it would be expected that they would be finally transformed into type I fibres in our model. Much to our surprise, the contrary occured, i.e. the muscle transformation was not stable : during the second month, type IIC fibres disappeared and, from then on, the histochemical muscle pattern did not differ from that observed after a single cold injury.

To explain this contradiction, we assumed that, since type IIC fibres have usually been considered as an intermediate type, some type II muscle fibres must have been reinnervated by type I axons and then temporarily converted into type IIC fibres before being " recaptured " by their native axons (type II axons). Moreover, our assumption was supported by the observation, after silver impregnation, of several axonal sprouts converging on the same motor endplate (fig. 2 d).

This hypothesis would imply multiple and polyneuronal innervation of type IIC fibres. However, although our morphological data pointed in this direction, they were not sufficient to conclude in favour of this mode of innervation, which has only been demonstrated by intracellular recording of multiple endplate potentials between 20 and 35 days after the third and final freezing of the sciatic nerve.

Therefore, our histological, cytochemical and electrophysiological observations can be explained by the transitory nature of the type IIC fibres and their multiple innervation. Thus, it may be assumed that type I axons were the first to regenerate and reinnervate both their native type I muscle fibres and certain contiguous type II (A or B) fibres which would then have become type IIC fibres. Similarly, type II axons may be expected to regenerate a little later and compete with foreign axons to recapture their native type II muscle fibres. The multiple innervation stage we observed obviously corresponds to this period of competition between two or several axons of different origins. In that case, all the muscle fibres should have recovered their native cytochemical type three months after the final freezing.

Our results on multiple innervation agree with the electrophysiological data obtained by others after partial (Brown and Ironton, 1978 ; Thompson, 1978) or total (Benoit and Changeux, 1978 ; Gorio et al., 1980 ; Jansen and Van Essen, 
1975 ; McArdle, 1975) denervation of mammalian skeletal muscles. However, our combined cytological, cytochemical and electrophysiological results provide evidence for the polyneuronal character of this type of innervation.

7e Réunion du groupe Développement I.N.R.A., Nouzilly/Tours, 14-15 mai 1981.

Résumé. De très nombreuses fibres IIC apparaissent dans le muscle gastrocnémien interne de rat adulte après plusieurs épisodes de dénervation-réinnervation provoqués par des congélations localisées itératives du nerf sciatique. L'ensemble de nos données cytologiques, cytochimiques et électrophysiologiques nous permettent raisonnablement de conclure que ces fibres IIC, inhabituelles dans le muscle normal, ont une innervation multiple et polyneuronale.

\section{References}

BENOIT P., CHANGEUX J. P., 1978. Consequences of blocking the nerve with local anaesthetic on the evolution of multinnervation of the regenerating neuromuscular junctions of the rat. Brain Res., 149, 89-96.

BROOKE M. H., KAISER K. K., 1970. Muscle fiber types : how many and what kind? Arch. Neurol., 23, 369-379.

BROOKE M. H., WILLIAMSON E. K., KAISER K. K., 1971. The behavior of four fiber types in developing and reinnervated muscle. Arch. Neurol., 25, 360-366.

BROWN M. C., IRONTON R., 1978. Sprouting and regression on neuromuscular synapses in partially denervated mammalian muscles. J. Physiol. (London), 278, 325-348.

BURKE R. E., LEVINE D. N., ZAJAC F. E., TSAIRIS P., ENGEL W. K., 1971. Direct correlation of physiological and histochemical characteristics in motor units of cat triceps surae muscles, 23-30. In DESMEDT J. E., New developments in electromyography and cfinical neuropathology, S. Karger, Basel.

EDSTRÖM L., KUGELBERG E., 1968. Histochemical composition, distribution of fibres and fatigability of single motor units. Anterior tibial muscles of the rat. J. Neurol. Neurosurg. Psychiat., 31, 424-433.

FARDEAU M., 1973. Caractéristiques cytochimiques et ultrastructurales des différents types de fibres musculaires squelettiques extra-fusales (chez l'Homme et quelques Mammifères). Ann. Anat. pathol., 18, 7-34.

GORIO A., CARMIGNOTO G., FACCI L., FINESSO M., 1980. Motor nerve sprouting induced by ganglioside treatment. Possible implications for gangliosides on neuronal growth. Brain Res., 197, 236-241.

JANSEN J. K. S., VAN ESSEN D. C., 1975. Reinnervation of rat skeletal muscle in the presence of $\alpha$-bungarotoxin. J. Physiol. (London), 250, 651-667.

KARPATI G., ENGEL W. K., 1968. "Type-grouping 》 in skeletal muscles after experimental reinnervation. Neurology, 18, 447-455.

McARDLE J. J., 1975. Complex endplate potentials at the regenerating neuromuscular junction of the rat. Exp. Neurol., 49, 629-638.

MIRA J. C., 1971. Maintien de la continuité de la lame basale des fibres nerveuses périphériques après "section" des axones par congélation localisée. C. R. Acad. Sci. Paris, Sér. D, 273, $1836-1839$.

MIRA J. C., 1979. Quantitative studies of the regeneration of rat myelinated fibres : variations in the number and size of regenerating nerve fibres after repeated localized freezings. J. Anat., 129, 77-93.

MIRA J. C., FARDEAU M., 1978 a. Modifications dans la distribution des types de fibres musculaires provoquées par des congélations localisées itératives du nerf sciatique de rat. $C . R$. Acad. Sci. Paris, Sér. D, 286, 1367-1370. 
MIRA J. C., FARDEAU M., 1978 b. Nerve and muscle changes induced by repeated localized freezings of the sciatic nerve in the rat, 83-90. In CANAL N., POZZA G., Peripheral neuropathies, Elsevier, Amsterdam.

MIRA J. C., PECOT-DECHAVASSINE M., 1978. Mise en évidence d'une innervation multiple des fibres musculaires squelettiques de rat à la suite de congélations localisées itératives du nerf sciatique. C. R. Soc. Biol., 172, 1063-1068.

MIRA J. C., PECOT-DECHAVASSINE M., 1979. Induction of multiple and polyneuronal innervation in skeletal muscle fibres after repeated localized freezings of the adult rat sciatic nerve. Cryoletters, 1, 5-10.

THOMPSON W., 1978. Reinnervation of partially denervated rat soleus muscle. Acta physiol. scand., 103, 81-91. 